Article

\title{
Effect of Feeding Discarded Durian Peel Ensiled with Lactobacillus casei TH14 and Additives in Total Mixed Rations on Digestibility, Ruminal Fermentation, Methane Mitigation, and Nitrogen Balance of Thai Native-Anglo-Nubian Goats
}

\author{
Natcha Panyawoot ${ }^{1} \mathbb{D}$, Sarong So ${ }^{2} \mathbb{D}$, Anusorn Cherdthong ${ }^{2} \mathbb{D}$ and Pin Chanjula ${ }^{1, *(\mathbb{D})}$ \\ 1 Animal Production Innovation and Management Division, Faculty of Natural Resources, Hat Yai Campus, \\ Prince of Songkla University, Songkhla 90112, Thailand; natcha9541@gmail.com \\ 2 Tropical Feed Resource Research and Development Center (TROFREC), Department of Animal Science, \\ Faculty of Agriculture, Khon Kaen University, Khon Kaen 40002, Thailand; sarong07so@gmail.com (S.S.); \\ anusornc@kku.ac.th (A.C.) \\ * Correspondence: pin.c@psu.ac.th; Tel.: +66-74-558-805; Fax: +66-74-558-803
}

check for updates

Citation: Panyawoot, N.; So, S.; Cherdthong, A.; Chanjula, P. Effect of Feeding Discarded Durian Peel Ensiled with Lactobacillus casei TH14 and Additives in Total Mixed Rations on Digestibility, Ruminal Fermentation, Methane Mitigation, and Nitrogen Balance of Thai Native-Anglo-Nubian Goats.

Fermentation 2022, 8, 43.

https://doi.org/10.3390/

fermentation 8020043

Academic Editor: Christian Kennes

Received: 26 December 2021

Accepted: 18 January 2022

Published: 21 January 2022

Publisher's Note: MDPI stays neutral with regard to jurisdictional claims in published maps and institutional affiliations.

Copyright: (c) 2022 by the authors. Licensee MDPI, Basel, Switzerland. This article is an open access article distributed under the terms and conditions of the Creative Commons Attribution (CC BY) license (https:/ / creativecommons.org/licenses/by/ $4.0 /)$.

\begin{abstract}
The objective of this study was to evaluate the effect of fermented discarded durian peel with Lactobacillus casei TH14, cellulase, and molasses separately or in combination in total mixed rations on feed utilization, digestibility, ruminal fermentation, and nitrogen utilization in growing crossbreed Thai Native-Anglo-Nubian goats. Five crossbreed Thai Native-Anglo-Nubian goats $(50 \%)$ at 9 to 12 months of age and $20 \pm 1$ of body weight (BW) were assigned to a $5 \times 5$ Latin square design. Evaluated treatments were fermented discarded durian peel without additives (FDP), fermented discarded durian peel with $5 \%$ of molasses (FDPM), fermented discarded durian peel with $2 \%$ of cellulase (FDPC), fermented discarded durian peel with $1.0 \times 10^{5} \mathrm{cfu} / \mathrm{g}$ fresh matter of $L$. casei TH14 (FDPL), and fermented discarded durian peel with $5 \%$ of molasses and $1.0 \times 10^{5} \mathrm{cfu} / \mathrm{g}$ fresh matter of L. casei TH14 (FDPML). This study showed that acid detergent fiber intake was different $(p<0.05)$ between goats fed FDP and those fed FDPLM, $0.24 \mathrm{~g} / \mathrm{d}$ and $0.20 \mathrm{~g} / \mathrm{d}$, respectively. The FDPML ration had significantly $(p<0.05)$ greater apparent nutrient digestibility and a better propionate concentration compared with other treatments. FDPML treatment significantly $(p<0.05)$ decreased the acetate-to-propionate ratio, methane production, and urinary nitrogen. Therefore, treated discarded durian peel with molasses and L. casei TH14 in combination could add $25 \%$ of dry matter into the diet for growing goats without a negative impact.
\end{abstract}

Keywords: goat feeding; durian peel; silage additives; propionate; methane mitigation; nitrogen balance

\section{Introduction}

Durian, a seasonal fruit, is grown widely in tropical countries, where Malaysia and Thailand are the main producers [1]. Approximately 20 to 30\% of durian is appropriate for human consumption, and 80 to $70 \%$ accounts for the durian peel, which is discarded as waste [2]. Discarded durian peel (DP) contains $10.30 \%$ crude protein (CP), $3.24 \%$ fat, $22.33 \%$ crude fiber (CF), 50.51\% nitrogen-free extract (NFE), 9.50\% cellulose, and 10.32\% acid detergent lignin (ADL) [3]. Due to a high NFE content, DP spoils shortly after discarding. Ensiling is a well-known technique and is used to preserve high-fermentable-containing feed resources using lactic acid bacteria (LAB), converting sugar into lactic acid, resulting in low pH [4]. Ensiling additives including Lactobacillus strains, cellulase, and molasses are usually added to improve fermentation quality [5-8]. Lactobacillus casei TH14 (L. casei TH14), LAB strain, is a local strain isolated from sweet corn silage, which has high lactic acid production with a low $\mathrm{pH}$ range [9]. Cellulase is a popular fibrolytic enzyme added to break down cellulose, releasing soluble carbohydrate for LAB growth [10,11], while 
molasses is added as a carbon source for LAB to ensure adequate lactic acid production if ensiling materials contain low water-soluble carbohydrate numbers [5]. Using L. casei TH14, cellulase and molasses have been reported to improve quality of sorghum [4], rice straw [12], and sugarcane bagasse [5]. In addition to fermentation quality improvement, L. casei TH14, cellulase, and molasses addition also improves feed utilization, propionate production, and methane mitigation $[7,8,13]$. However, the effect of L. casei TH14, cellulase, and molasses on DP quality and using fermented DP as roughage source in goat rations have never been evaluated. This study hypothesized that L. casei TH14 combined with molasses could improve DP quality, nutrient digestibility, propionate production, and methane mitigation. The objective of this study was to evaluate the effect of fermented discarded durian peel with Lactobacillus casei TH14, cellulase, and molasses separately or in combination in total mixed rations on feed utilization, digestibility, ruminal fermentation, and nitrogen utilization in growing crossbreed Thai Native-Anglo-Nubian goats.

\section{Materials and Methods}

\subsection{Animal Ethics}

The use of goats in this study was approved (MHESI 68014/674) by Institutional Animal Care and Use Committee, Prince of Songkla University.

\subsection{Animals and Experimental Design}

Five crossbreed Thai Native-Anglo-Nubian goats (50\%) at 9 to 12 months of age and $20 \pm 1$ of body weight (BW) were used in this study. All goats were injected with ivermectin (IDECTIN $^{\circledR}$ The British Dispensary (L.P.) CO., Ltd., Bangkok, Thailand) with $1 \mathrm{~mL}$ dose per $50 \mathrm{~kg}$ of $\mathrm{BW}$ to kill parasites before starting the experiment. Goats were assigned to a $5 \times 5$ Latin square design. Treatments were fermented discarded durian peel without additives (FDP), fermented discarded durian peel with $5 \%$ of molasses (FDPM), fermented discarded durian peel with $2 \%$ of cellulase (FDPC), fermented discarded durian peel with $1.0 \times 10^{5} \mathrm{cfu} / \mathrm{g}$ fresh matter of L. casei TH14 (FDPL), and fermented discarded durian peel with $5 \%$ of molasses, and $1.0 \times 10^{5} \mathrm{cfu} / \mathrm{g}$ fresh matter of L. casei TH14 (FDPML).

\subsection{Fermented Discarded Durian Peel Preparation}

Discarded durian peel (Monthong-Durio zibthinus Murray) was obtained from Seahorse Intertrade Company Limited in Chana District, Songkhla Province, Thailand, and cut into 1 to $2 \mathrm{~cm}$ pieces. Then, discarded durian peel was fermented with the respective additives including molasses at 5\% [5], cellulase at $2 \%$ [14], and L. casei TH14 at $1.0 \times 10^{5} \mathrm{cfu} / \mathrm{g}$ fresh matter [4]. Cellulase (powder form, $5 \times 10^{5} \mathrm{U} / \mathrm{g}$ activity, CAS number: 9004-34-6, Sinobios Imp. \& Exp., Thanghai, China) and L. casei TH14 as a silage starter (composed of $80 \%$ trehalose, $15 \%$ lactose, and $1.0 \times 10^{11} \mathrm{cfu} / \mathrm{g}$ L. casei TH14; Bio Ag Khon Kaen, Khon Kaen, Thailand) were used. Molasses was purchased from a local supplier located in Hat Yai District, Songkla Province, Thailand. Additives were dissolved in clean water, sprayed on discarded durian peel, mixed well, and fermented in $50 \mathrm{~L}$ plastic buckets (Changzhou Treering Plastics Co., Ltd., Changzhou, China) for 30 days. After fermenting for 30 days, fermented discarded durian peel samples were collected, dried at $60^{\circ} \mathrm{C}$ for $72 \mathrm{~h}$, and ground into $1 \mathrm{~mm}$ pieces to analyze the dry matter (DM), CP, and ash according to AOAC [15], and neutral detergent fiber (NDF), acid detergent fiber (ADF), and acid detergent lignin according to Van Soest et al. [16]. The chemical composition of fermented discarded durian peel is provided under Table 1. Fermentation characteristics of fermented DP were assessed. $\mathrm{pH}$ was measured according to Chen et al. [14] using $\mathrm{pH}$ meter (HANNA instruments HI 98153 microcomputer $\mathrm{pH}$ meter, Kallang Avenue, Singapore); briefly, $20 \mathrm{~g}$ of fermented DP samples were taken and mixed with $80 \mathrm{~mL}$ of distilled water and kept at $10{ }^{\circ} \mathrm{C}$ for $24 \mathrm{~h}$. Samples were prepared with ammonia nitrogen $\left(\mathrm{NH}_{3}-\mathrm{N}\right)$ using spectrophotometer (UV/VIS Spectrometer, PG Instruments Ltd., London, UK) and volatile fatty acids (lactic acid, acetate, and butyrate) using gas chromatography and analyzed according to So 
et al. [8] The $\mathrm{pH}, \mathrm{NH}_{3}-\mathrm{N}$, lactate, acetate, and butyrate of fermented discarded durian peel are provided in Table 1.

Table 1. Nutrient composition of TMR diets, rice straw, and fermented discarded durian peel quality treated with or without additives.

\begin{tabular}{|c|c|c|c|c|c|c|}
\hline \multirow{2}{*}{$\begin{array}{l}\text { Nutrient Composition } \\
\text { (\% of DM) }\end{array}$} & \multicolumn{5}{|c|}{ Treatments } & \multirow{2}{*}{ Rice Stran } \\
\hline & FDP & FDPM & FDPC & FDPL & FDPML & \\
\hline DM & 42.50 & 42.72 & 37.20 & 37.77 & 37.53 & 92.12 \\
\hline $\mathrm{OM}$ & 93.27 & 93.26 & 93.09 & 93.28 & 93.96 & 91.80 \\
\hline $\mathrm{CP}$ & 15.51 & 15.39 & 15.80 & 15.39 & 15.69 & 2.81 \\
\hline $\mathrm{NFC}+$ & 10.82 & 12.30 & 19.55 & 17.21 & 21.49 & 12.72 \\
\hline $\mathrm{NDF}$ & 65.45 & 63.12 & 57.26 & 57.30 & 57.01 & 74.71 \\
\hline $\mathrm{ADF}$ & 29.75 & 28.51 & 28.28 & 27.41 & 27.22 & 56.55 \\
\hline $\mathrm{ADL}$ & 8.52 & 7.98 & 8.26 & 6.74 & 6.59 & 4.59 \\
\hline GE kcal/kg DM & 4361.00 & 4306.26 & 4315.18 & 4363.60 & 4315.40 & 3501.53 \\
\hline \multicolumn{7}{|c|}{ Fermented discarded durian peel quality } \\
\hline $\mathrm{DM}, \%$ & 17.0 & 17.3 & 16.5 & 16.5 & 17.4 & \\
\hline $\mathrm{OM}, \%$ of $\mathrm{DM}$ & 94.0 & 93.4 & 92.9 & 93.7 & 93.4 & \\
\hline $\mathrm{CP}, \%$ of $\mathrm{DM}$ & 7.3 & 8.3 & 7.2 & 7.2 & 7.5 & \\
\hline NDF, $\%$ of DM & 62.5 & 61.0 & 73.4 & 61.9 & 65.5 & \\
\hline $\mathrm{ADF}, \%$ of $\mathrm{DM}$ & 41.7 & 38.6 & 45.8 & 43.9 & 42.3 & \\
\hline $\mathrm{GE}, \mathrm{kcal} / \mathrm{kg} \mathrm{DM}$ & 4413.8 & 4314.7 & 4737.4 & 4449.1 & 4205.4 & \\
\hline $\mathrm{pH}$ & 3.74 & 3.79 & 3.66 & 3.73 & 3.74 & \\
\hline $\mathrm{NH}_{3}-\mathrm{N}, \%$ of $\mathrm{CP}$ & 1.29 & 0.70 & 1.18 & 0.74 & 0.89 & \\
\hline Lactic acid, $\%$ of DM & 10.12 & 10.79 & 10.89 & 10.54 & 10.98 & \\
\hline Acetic acid, $\%$ of DM & 1.02 & 1.05 & 1.07 & 1.04 & 1.09 & \\
\hline Butyric acid, $\%$ of DM & 1.32 & 1.30 & 1.37 & 1.29 & 1.28 & \\
\hline
\end{tabular}

FDP $=$ untreated discarded durian peel in TMR; FDPM = treated discarded durian peel with molasses in TMR FDPC $=$ treated discarded durian peel with cellulase in TMR; FDPL $=$ treated discarded durian peel with $L$. casel TH14 in TMR; FDPML = treated discarded durian peel with molasses and L. casei TH14 in TMR. TMR compositions contain $25 \%$ fermented discarded durian peel with or without additives, $15 \%$ rice straw, $35.8 \%$ ground corn, $7.9 \%$ soybean meal, $0.4 \%$ fish meal, $5.4 \%$ leucaena meal, $7.2 \%$ palm kernel cake, $2.2 \%$ molasses, $0.3 \%$ dicalcium phosphate, $0.2 \%$ salt, and $0.6 \%$ premix. Premix per kg contains vitamin A: 10,000,000 IU; vitamin E: 70,000 IU, vitamin D: 1,600,000 IU; Fe: 50 g; Zn: 40 g; Mn: 40 g; Co: 0.1 g; Cu: 10 g; Se: 0.1 g; and I: 0.5 g. DM = dry matter; $\mathrm{OM}=$ organic matter; $\mathrm{CP}=$ crude protein; $\mathrm{NFC}=$ non-fiber carbohydrate; $\mathrm{NDF}=$ neutral detergent fiber; $\mathrm{ADF}=$ acid detergent fiber; $\mathrm{ADL}=$ acid detergent lignin; $\mathrm{GE}=$ gross energy. ${ }^{\dagger} \mathrm{NFC}=100-(\% \mathrm{NDF}+\% \mathrm{CP}+\%$ $\mathrm{EE}+\%$ ash) [17].

\subsection{Feeding, Sample Collection, and Analysis}

Feeding trial consisted of five 21-day periods, in which 14 days were used for dietary treatment adaptation and 7 days were used for sample analysis. Goats were separately stored in pens $(0.11 \times 0.95 \mathrm{~m})$ with free access to clean water and mineral lick and fed daily ad libitum total mixed rations at a 40:60 ratio (25\% fermented discarded durian peel, $15 \%$ rice straw, and $60 \%$ concentrate) at 8:00 a.m. and 16:00 p.m. The diets were formulated to meet the nutrient requirements of goats according to NRC [18], and chemical composition of dietary treatments is provided in Table 1. Diets offered and refusal were recorded daily to calculate DM intake. Goats were weighed at the beginning of the trial and at the end of each period throughout the trial to adjust DM intake and calculate the BW change of goats at the end of the trial.

During the last 7 days of each period, goats were kept in metabolism crate for sample collection and digestibility study. Diet and refusal data were collected throughout 7 days and divided into two portions. The first portion was used to analyze for DM content using oven drying at $100^{\circ} \mathrm{C}$, and second portion was deposited according to goat and period and stored at $-20^{\circ} \mathrm{C}$ for chemical composition analysis. Fecal and urine samples were gathered using total collection procedure. A total of $200 \mathrm{~g}$ of fecal sample was collected and oven-dried at $100{ }^{\circ} \mathrm{C}$ for DM analysis, and $5 \%$ of total feces was collected, deposited according to goat and period, and stored at $-20^{\circ} \mathrm{C}$ until analysis. Urine yield was collected using $5 \mathrm{~L}$ plastic tank consisting of $1 \mathrm{M} \mathrm{H}_{2} \mathrm{SO}_{4}$ to prevent nitrogen loss, and $10 \%$ of total 
urine was taken, deposited according to goat and period, and stored at $-20^{\circ} \mathrm{C}$. Before analysis, diet, refusal, and fecal samples were thawed, oven-dried at $60{ }^{\circ} \mathrm{C}$ for $72 \mathrm{~h}$, and ground through a $1 \mathrm{~mm}$ screen to analyze for DM, CP, and OM using AOAC [15]. The NDF, ADF and ADL content were analyzed using Ankom fiber analyzer according to Van Soest et al. [16]. Gross energy content in diet, refusal, and fecal samples was analyzed using Bomb calorimetry (LECO, Berrien, MI, USA). Urine samples were thawed and analyzed for nitrogen content using AOAC [15] method to study nitrogen balance.

On day 21 of each period, at $0 \mathrm{~h}$ before feeding and $4 \mathrm{~h}$ after feeding, approximately $100 \mathrm{~mL}$ of ruminal fluid was collected using vacuum pump attached with stomach tube. Ruminal pH measurement was conducted immediately using $\mathrm{pH}$ meter (HANNA instruments HI 98153 microcomputer pH meter, Kallang Avenue, Singapore). Then, $60 \mathrm{~mL}$ of ruminal fluid samples was kept in plastic bottle containing $1 \mathrm{M} \mathrm{H}_{2} \mathrm{SO}_{4}$ at a ratio 1:10 $(1 \mathrm{~mL}$ of $\mathrm{H}_{2} \mathrm{SO}_{4}$ : $10 \mathrm{~mL}$ of ruminal fluid) and centrifuged at $3000 \times \mathrm{g}$ for $15 \mathrm{~min}$. Approximately $35 \mathrm{~mL}$ of supernatant was taken and stored at $-20{ }^{\circ} \mathrm{C}$ to analyze $\mathrm{NH}_{3}-\mathrm{N}$ using Kjeltec Auto 2200 analyzer (Foss, Tecator, UK) according to Bremner and Keeney [19] and volatile fatty acid including acetate, propionate, and butyrate were analyzed using gas chromatography (model HP6890, Hewlett-Packard, Palo Alto, CA, USA; column: Restek 1207384, Stabilwax $\left.-60{ }^{\circ} \mathrm{C}-250{ }^{\circ} \mathrm{C}, 30 \mathrm{~m} \times 250 \mu \mathrm{m} \times 0.25 \mu \mathrm{m}\right)$ according to Osaki et al. [20] as described by So et al. [8] Methane $\left(\mathrm{CH}_{4}\right)$ production was estimated using Moss et al. [21] equation, $\mathrm{CH}_{4}$ $(\mathrm{g} / \mathrm{d})=0.45 \times$ acetate $-0.275 \times$ propionate $+0.4 \times$ butyrate. Another $1 \mathrm{~mL}$ of ruminal fluid sample was kept in plastic bottle consisting of $9 \mathrm{~mL}$ of $10 \%$ formalin and stored in $4{ }^{\circ} \mathrm{C}$ in a refrigerator to count bacteria, protozoa, and fungi population using total direct count technique according to Galyean [22].

On day 21 of each period, samples of approximately $3 \mathrm{~mL}$ of blood were collected from the jugular vein at $0 \mathrm{~h}$ before feeding and $4 \mathrm{~h}$ after feeding and placed in heparinized tubes. Then, blood samples were centrifuged at $3000 \times g$ for $10 \mathrm{~min}$, and plasma samples were taken and stored at $-20{ }^{\circ} \mathrm{C}$ until analysis. Plasma samples were sent to Stanbio Laboratory (An EKF Diagnostics Company, Boerne, TX, USA) and used to analyze blood urea nitrogen (procedure no. 2020), glucose (procedure no. 1070), total protein (procedure no. 0250), and albumin (procedure no. 0285). Pack cell volume was measured using micro-hematocrit method, and mean corpuscular hemoglobin concentration, globulin, and albumin to globulin ratio were obtained by calculation. Hemoglobin was measured using commercial kits (Diamond Diagnostics, Egypt). Red blood cell count, mean corpuscular volume, and RBC distribution width were measured using hematological analyzer (ABX Micros 60, HORBA ABX, France).

\subsection{Statistical Analysis}

All data were analyzed using Proc Mixed model of SAS as follows:

$$
Y_{i j k l}=\mu+\rho_{i}+\gamma_{j}+t_{1}+\tau_{k}+\varepsilon_{i j k l}
$$

where $Y_{i j k l}$ are the observation parameters, $\mu$ is the overall mean, $\rho_{i}$ is the random effect of animal, $\gamma_{j}$ is the random effect of periods, $t_{1}$ is the random effect of time, $\tau_{k}$ is the fixed effect of treatments, and $\varepsilon_{\mathrm{ijkl}}$ is the error term. Differences among treatments were compared using Duncan's multiple range test, statistically accepted at $p<0.05$.

\section{Results}

\subsection{Nutrient Content of Diets}

Dietary treatments were formulated to have approximately 15\% CP content (Table 1). NFC, NDF, ADF, and ADL content varied among dietary treatments due to the quality of untreated and treated discarded durian peel used in the formulation. The diet containing untreated discarded durian peel (FDP) showed the highest NDF, ADF, and ADLs content and the lowest NFC content compared with FDPM, FDPC, FDPL, and FDPML diets. The chemical composition of untreated and treated discarded durian peel is provided (footnote of Table 1). 


\subsection{Nutrient Intake and Body Weight Change}

Table 2 presents the intake of DM, OM, CP, NDF, and ADF, weight gain, and BW change in growing goats fed TMR containing untreated and treated discarded durian peel with additives. DM, OM, CP, and NDF intake were not different among treatments except ADF. ADF intake was different $(p<0.05)$ between FDP and FDPLM, which contained $0.24 \mathrm{~g} / \mathrm{d}$ and $0.20 \mathrm{~g} / \mathrm{d}$, respectively. Weight gain and BW change in goats were not affected $(p>0.05)$ by dietary treatments.

Table 2. Effects of untreated and treated discarded durian peel in TMR on intake and body weight change in growing goats.

\begin{tabular}{|c|c|c|c|c|c|c|c|}
\hline \multirow{2}{*}{ Items } & \multicolumn{5}{|c|}{ Dietary Treatments } & \multirow{2}{*}{ SEM } & \multirow{2}{*}{$p$-Value } \\
\hline & FDP & FDPM & FDPC & FDPL & FDPML & & \\
\hline \multicolumn{8}{|l|}{ DM intake } \\
\hline $\mathrm{kg} / \mathrm{d}$ & 0.796 & 0.757 & 0.785 & 0.797 & 0.806 & 0.02 & 0.56 \\
\hline$\%$ BW & 2.96 & 2.84 & 2.89 & 2.94 & 3.04 & 0.06 & 0.28 \\
\hline $\mathrm{g} / \mathrm{kg} \mathrm{BW} 0.75$ & 67.39 & 64.44 & 66.08 & 67.66 & 68.98 & 1.56 & 0.35 \\
\hline \multicolumn{8}{|c|}{ Nutrient intake, g/d } \\
\hline $\mathrm{OM}$ & 0.752 & 0.696 & 0.711 & 0.744 & 0.712 & 0.03 & 0.54 \\
\hline $\mathrm{CP}$ & 0.125 & 0.115 & 0.120 & 0.123 & 0.119 & 0.01 & 0.42 \\
\hline NDF & 0.528 & 0.471 & 0.437 & 0.457 & 0.432 & 0.02 & 0.05 \\
\hline $\mathrm{ADF}$ & $0.236^{\mathrm{a}}$ & $0.215^{a b}$ & $0.216^{\mathrm{ab}}$ & $0.216^{\mathrm{ab}}$ & $0.199 \mathrm{~b}$ & 0.01 & 0.04 \\
\hline Weight gain, kg & 2.40 & 1.90 & 1.90 & 2.80 & 2.30 & 0.38 & 0.45 \\
\hline BW change, $\mathrm{kg} / \mathrm{d}$ & 0.114 & 0.090 & 0.090 & 0.132 & 0.110 & 0.01 & 0.46 \\
\hline BW change, $\%$ & 9.17 & 7.61 & 7.12 & 10.70 & 9.00 & 1.36 & 0.41 \\
\hline
\end{tabular}

$\mathrm{SEM}$ = standard error of the mean; $\mathrm{BW}^{0.75}=$ metabolic body weight; $\mathrm{DM}=$ dry matter, $\mathrm{OM}=$ organic matter; $\mathrm{CP}=$ crude protein; $\mathrm{NDF}=$ neutral detergent fiber; $\mathrm{ADF}=$ acid detergent fiber; $\mathrm{BW}=$ body weight. ${ }^{\mathrm{a}, \mathrm{b}}$ Means in the same row with different letters differ $(p<0.05)$.

\subsection{Apparent Nutrient Digestibility, Digestible Nutrient Intake, and Energy Intake}

Apparent total tract digestibility was affected significantly $(p<0.05)$ by dietary treatments (Table 3). DM, OM, CP, NDF, and ADF digestibility were significantly lower in FDP compared with FDPML. FDPM, FDPC, FDPL, and FDPML were comparable in terms of DM, OM, CP, NDF, and ADF digestibility. Digestible nutrient intake including OM, $\mathrm{CP}, \mathrm{NDF}$, and ADF was not different $(p>0.05)$ among treatments. Estimated ME intake, expressed as Mcal/d, was not significant among treatments; however, estimated ME intake, expressed as per $\mathrm{kg}$ DM intake, was significant $(p<0.05)$ among treatments. ME intake, expressed as per kg DM intake, was significantly observed between FDP, FDPM and FDPML, in amounts of $2.55 \mathrm{Mcal} / \mathrm{kg} \mathrm{DM}, 2.62 \mathrm{Mcal} / \mathrm{kg} \mathrm{DM}$, and 2.69 Mcal/kg DM, respectively. 
Table 3. Effects of untreated and treated discarded durian peel in TMR on nutrient digestibility and digestible nutrient intake of growing goats.

\begin{tabular}{|c|c|c|c|c|c|c|c|}
\hline \multirow{2}{*}{ Items } & \multicolumn{5}{|c|}{ Dietary Treatments } & \multirow{2}{*}{ SEM } & \multirow{2}{*}{$p$-Value } \\
\hline & FDP & FDPM & FDPC & FDPL & FDPML & & \\
\hline \multicolumn{8}{|l|}{$\begin{array}{l}\text { Apparent total } \\
\text { tract digestibility, } \\
\%\end{array}$} \\
\hline $\mathrm{DM}$ & $70.42^{b}$ & $72.79^{a}$ & $73.91^{\mathrm{a}}$ & $74.07^{\mathrm{a}}$ & $73.81^{\mathrm{a}}$ & 0.45 & $<0.01$ \\
\hline OM & $71.82^{b}$ & $73.95^{a}$ & $75.02^{a}$ & $75.48^{a}$ & $75.16^{\mathrm{a}}$ & 0.49 & $<0.01$ \\
\hline $\mathrm{CP}$ & $68.42^{c}$ & $71.11^{\mathrm{b}}$ & $72.66^{a b}$ & $73.07^{a b}$ & $73.63^{a}$ & 0.61 & $<0.01$ \\
\hline NDF & $63.83^{b}$ & $70.48^{a}$ & $70.23^{a}$ & $70.96^{a}$ & $71.03^{a}$ & 0.47 & $<0.01$ \\
\hline $\mathrm{ADF}$ & $40.96^{b}$ & $47.13^{\mathrm{a}}$ & $47.09^{\mathrm{a}}$ & $47.10^{\mathrm{a}}$ & $46.08^{a}$ & 0.96 & $<0.01$ \\
\hline \multicolumn{8}{|l|}{$\begin{array}{l}\text { Digestible nutrient } \\
\text { intake, } \mathrm{kg} / \mathrm{d}\end{array}$} \\
\hline $\mathrm{OM}$ & 0.543 & 0.515 & 0.534 & 0.562 & 0.536 & 0.02 & 0.47 \\
\hline $\mathrm{CP}$ & 0.087 & 0.084 & 0.086 & 0.090 & 0.087 & 0.01 & 0.68 \\
\hline NDF & 0.338 & 0.332 & 0.307 & 0.325 & 0.307 & 0.01 & 0.21 \\
\hline $\mathrm{ADF}$ & 0.098 & 0.102 & 0.102 & 0.102 & 0.092 & $<0.01$ & 0.38 \\
\hline \multicolumn{8}{|l|}{$\begin{array}{c}\text { Estimated energy } \\
\text { intake }\end{array}$} \\
\hline ME Mcal/d & 2.06 & 1.96 & 2.03 & 2.14 & 2.04 & 0.07 & 0.48 \\
\hline ME Mcal/kg DM & $2.55^{\mathrm{c}}$ & $2.62^{b}$ & $2.65^{\mathrm{ab}}$ & $2.68^{a b}$ & $2.69^{a}$ & 0.02 & $<0.01$ \\
\hline
\end{tabular}

FDP $=$ untreated discarded durian peel $;$ FDPM $=$ treated discarded durian peel with molasses; FDPC $=$ treated discarded durian peel with cellulase; FDPL $=$ treated discarded durian peel with $L$. casei $T H 14 ;$ FDPML $=$ treated discarded durian peel with molasses and L. casei TH14; SEM = standard error of the mean; DM = dry matter $\mathrm{OM}=$ organic matter; $\mathrm{CP}=$ crude protein $; \mathrm{NDF}=$ neutral detergent fiber; $\mathrm{ADF}=$ acid detergent fiber; $\mathrm{ME}=$ metabolizable energy. ${ }^{\text {a-c }}$ Means in the same row with different letters differ $(p<0.05) .{ }^{\dagger} 1 \mathrm{~kg}$ DOM $=3.8 \mathrm{Mcal}$ $\mathrm{ME} / \mathrm{kg}$ [23].

\subsection{Rumen Fermentation Characteristics}

Dietary treatments significantly affected ruminal $\mathrm{pH}$ and $\mathrm{NH}_{3}-\mathrm{N}$ but not ruminal temperature (Table 4). Mean ruminal $\mathrm{pH}$ and $\mathrm{NH}_{3}-\mathrm{N}$ were significantly $(p<0.05)$ observed between FDP and FDPML but FDPM, FDPC, FDPL, and FDPML were comparable. Ruminal $\mathrm{pH}$ and $\mathrm{NH}_{3}-\mathrm{N}$ were 6.71 and $22.58 \mathrm{mg} / \mathrm{dL}$ in FDP and 6.44 and $17.29 \mathrm{mg} / \mathrm{dL}$ in FDPML, respectively. Dietary treatments significantly affected propionate concentration, the acetateto-propionate ratio and acetate-butyrate-to-propionate ratio except total VFA, acetate, and butyrate concentration. The propionate concentration was significantly higher, while the acetate-to-propionate ratio and acetate- butyrate-to-propionate ratio were significantly lower in FDPML than FDP, FDPM, FDPC, and FDPL. FDPML showed the highest mean propionate concentration $(20.31 \%)$ and lowest mean acetate-to-propionate ratio (3.58) and acetate + butyrate-to-propionate ratio (3.94). $\mathrm{CH}_{4}$ production was affected significantly $(p<0.05)$ by dietary treatments. Mean $\mathrm{CH}_{4}$ production was significant between FDP and FDPML, at $32.93 \mathrm{~g} / \mathrm{d}$ and $29.41 \mathrm{~g} / \mathrm{d}$, respectively, but FDP, FDPM, FDPC, and FDPL were comparable for $\mathrm{CH}_{4}$ production. 
Table 4. Effects of untreated and treated discarded durian peel on rumen characteristics in growing goats.

\begin{tabular}{|c|c|c|c|c|c|c|c|}
\hline \multirow{2}{*}{ Items } & \multicolumn{5}{|c|}{ Dietary Treatments } & \multirow{2}{*}{ SEM } & \multirow{2}{*}{$p$-Value } \\
\hline & FDP & FDPM & FDPC & FDPL & FDPML & & \\
\hline \multicolumn{8}{|c|}{ Ruminal pH } \\
\hline $0 \mathrm{~h}$ & 6.61 & 6.56 & 6.55 & 6.56 & 6.50 & 0.09 & 0.94 \\
\hline $4 \mathrm{~h}$ & 6.81 & 6.64 & 6.39 & 6.57 & 6.39 & 0.11 & 0.06 \\
\hline Mean & $6.71^{\mathrm{a}}$ & $6.60^{a b}$ & $6.47^{\mathrm{b}}$ & $6.56^{\mathrm{ab}}$ & $6.44^{\mathrm{b}}$ & 0.05 & 0.04 \\
\hline \multicolumn{8}{|c|}{ Temperature, ${ }^{\circ} \mathrm{C}$} \\
\hline $0 \mathrm{~h}$ & 39.0 & 39.2 & 39.0 & 39.2 & 39.2 & 0.15 & 0.50 \\
\hline $4 \mathrm{~h}$ & 39.6 & 39.4 & 39.2 & 39.2 & 39.6 & 0.25 & 1.00 \\
\hline Mean & 39.3 & 39.3 & 39.1 & 39.2 & 39.4 & 0.19 & 0.78 \\
\hline \multicolumn{8}{|c|}{ Ammonia-nitrogen, $\mathrm{mg} / \mathrm{dL}$} \\
\hline $0 \mathrm{~h}$ & $22.29^{a}$ & $18.57^{\mathrm{b}}$ & $20.29 \mathrm{ab}$ & $19.14^{\mathrm{b}}$ & $18.00^{b}$ & 0.78 & 0.02 \\
\hline $4 \mathrm{~h}$ & $22.86^{\mathrm{a}}$ & $18.57^{\mathrm{b}}$ & $18.29^{b}$ & $18.57^{b}$ & $16.57^{b}$ & 0.63 & $<0.01$ \\
\hline Mean & $22.58^{a}$ & $18.57^{\mathrm{b}}$ & $19.29^{b}$ & $18.86^{b}$ & $17.29^{b}$ & 0.43 & $<0.01$ \\
\hline \multicolumn{8}{|c|}{ Total volatile fatty acid, $\mathrm{mM} / \mathrm{L}$} \\
\hline $0 \mathrm{~h}$ & 86.50 & 93.13 & 97.63 & 96.79 & 99.54 & 6.26 & 0.62 \\
\hline $4 \mathrm{~h}$ & 95.12 & 97.09 & 100.26 & 102.30 & 104.58 & 8.42 & 0.93 \\
\hline Mean & 90.81 & 95.11 & 98.95 & 99.55 & 102.06 & 4.98 & 0.56 \\
\hline \multicolumn{8}{|c|}{ Acetate, \% } \\
\hline $0 \mathrm{~h}$ & 74.17 & 77.22 & 75.94 & 74.31 & 71.73 & 1.49 & 0.17 \\
\hline $4 \mathrm{~h}$ & 73.99 & 73.77 & 72.31 & 72.89 & 70.75 & 1.14 & 0.32 \\
\hline Mean & 74.08 & 75.49 & 74.12 & 73.61 & 71.24 & 1.21 & 0.23 \\
\hline \multicolumn{8}{|c|}{ Propionate, $\%$} \\
\hline $0 \mathrm{~h}$ & $13.88^{b}$ & $13.23^{b}$ & $15.03^{b}$ & $15.82^{b}$ & $19.77^{\mathrm{a}}$ & 0.82 & $<0.01$ \\
\hline $4 \mathrm{~h}$ & $16.28^{b}$ & $16.83^{b}$ & $18.17^{b}$ & $17.92^{b}$ & $20.85^{a}$ & 0.73 & $<0.01$ \\
\hline Mean & $15.08^{b}$ & $15.03^{b}$ & $16.59^{b}$ & $16.87^{b}$ & $20.31^{a}$ & 0.66 & $<0.01$ \\
\hline \multicolumn{8}{|c|}{ Butyrate, \% } \\
\hline $0 \mathrm{~h}$ & 9.77 & 7.81 & 7.45 & 8.06 & 7.18 & 0.87 & 0.30 \\
\hline $4 \mathrm{~h}$ & 8.91 & 7.96 & 8.32 & 8.24 & 7.50 & 0.54 & 0.49 \\
\hline Mean & 9.34 & 7.89 & 7.89 & 8.15 & 7.34 & 0.64 & 0.31 \\
\hline \multicolumn{8}{|c|}{ Acetate:Propionate ratio } \\
\hline $0 \mathrm{~h}$ & $6.02^{\mathrm{a}}$ & $5.98^{\mathrm{a}}$ & $5.32^{\mathrm{a}}$ & $4.92^{\mathrm{a}}$ & $3.73^{b}$ & 0.38 & $<0.01$ \\
\hline $4 \mathrm{~h}$ & $4.62^{\mathrm{a}}$ & $4.49^{\mathrm{a}}$ & $4.16^{\mathrm{ab}}$ & $4.14^{\mathrm{ab}}$ & $3.42^{b}$ & 0.27 & 0.05 \\
\hline Mean & $5.32^{a}$ & $5.23^{\mathrm{a}}$ & $4.74^{\mathrm{a}}$ & $4.53^{\mathrm{a}}$ & $3.58^{b}$ & 0.27 & $<0.01$ \\
\hline \multicolumn{8}{|c|}{ Acetate + Butyrate:Propionate ratio } \\
\hline $0 \mathrm{~h}$ & $6.74^{\mathrm{a}}$ & $6.58^{\mathrm{a}}$ & $5.85^{a}$ & $5.45^{\mathrm{a}}$ & $4.11^{b}$ & 0.40 & $<0.01$ \\
\hline $4 \mathrm{~h}$ & $5.18^{a}$ & $4.96^{\mathrm{a}}$ & $4.62 \mathrm{ab}$ & $4.60^{\mathrm{ab}}$ & $3.78^{b}$ & 0.28 & 0.04 \\
\hline Mean & $5.96^{\mathrm{a}}$ & $5.77 \mathrm{ab}$ & $5.24^{\mathrm{ab}}$ & $5.03^{b}$ & $3.94^{\mathrm{c}}$ & 0.27 & $<0.01$ \\
\hline \multicolumn{8}{|c|}{ Methane, g/d } \\
\hline $0 \mathrm{~h}$ & $33.47^{\mathrm{a}}$ & $34.23^{\mathrm{a}}$ & $33.02^{\mathrm{a}}$ & $32.32^{\mathrm{a}}$ & $29.72^{b}$ & 0.65 & $<0.01$ \\
\hline $4 \mathrm{~h}$ & $32.38^{a}$ & $31.75^{\mathrm{a}}$ & $30.88^{a}$ & $31.17^{\mathrm{a}}$ & $29.10^{\mathrm{b}}$ & 0.56 & 0.01 \\
\hline Mean & $32.93^{a}$ & $32.99^{a}$ & $31.95^{\mathrm{a}}$ & $31.74^{\mathrm{a}}$ & $29.41^{b}$ & 0.52 & $<0.01$ \\
\hline
\end{tabular}

FDP $=$ untreated discarded durian peel; FDPM = treated discarded durian peel with molasses; FDPC = treated discarded durian peel with cellulase; FDPL = treated discarded durian peel with $L$. casei TH14; FDPML = treated discarded durian peel with molasses and L. casei TH14; SEM = standard error of the mean. ${ }^{\text {a-c }}$ Means in the same row with different letters differ $(p<0.05)$.

\subsection{Microbial Population}

Dietary treatments did not affect $(p>0.05)$ the bacteria, fungal zoospores, total protozoa, Holotrich sp., or Entodiniomorphs sp. populations (Table 5). The mean total protozoal population was lowest in FDPML, at $2.46 \times 10^{6}$ cell $/ \mathrm{mL}$. 
Table 5. Effect of untreated and treated discarded durian peel in TMR on ruminal microbe population in growing goats.

\begin{tabular}{|c|c|c|c|c|c|c|c|}
\hline \multirow{2}{*}{ Items } & \multicolumn{5}{|c|}{ Dietary Treatments } & \multirow{2}{*}{ SEM } & \multirow{2}{*}{$p$-Value } \\
\hline & FDP & FDPM & FDPC & FDPL & FDPML & & \\
\hline \multicolumn{8}{|c|}{ Bacteria, $\times 10^{10}$ cell $/ \mathrm{mL}$} \\
\hline $0 \mathrm{~h}$ & 1.60 & 1.56 & 1.45 & 1.35 & 1.45 & 1.38 & 0.50 \\
\hline $4 \mathrm{~h}$ & 1.90 & 2.20 & 1.67 & 1.63 & 1.56 & 2.01 & 0.67 \\
\hline Mean & 1.75 & 1.88 & 1.56 & 1.49 & 1.51 & 1.67 & 0.43 \\
\hline \multicolumn{8}{|c|}{ Fungal zoospores, $\times 10^{6} \mathrm{cell} / \mathrm{mL}$} \\
\hline $0 \mathrm{~h}$ & 2.18 & 1.92 & 1.67 & 1.67 & 1.55 & 0.29 & 0.13 \\
\hline $4 \mathrm{~h}$ & 2.25 & 2.67 & 2.16 & 1.65 & 1.62 & 0.40 & 0.19 \\
\hline Mean & 2.21 & 2.29 & 1.92 & 1.66 & 1.59 & 0.30 & 0.15 \\
\hline \multicolumn{8}{|c|}{ Total Protozoa, $\times 10^{6} \mathrm{cell} / \mathrm{mL}$} \\
\hline $0 \mathrm{~h}$ & 2.88 & 2.51 & 2.47 & 2.21 & 2.29 & 0.26 & 0.11 \\
\hline $4 \mathrm{~h}$ & 3.16 & 3.47 & 3.15 & 2.63 & 2.61 & 0.32 & 0.13 \\
\hline Mean & 3.02 & 2.99 & 2.81 & 2.41 & 2.46 & 0.26 & 0.09 \\
\hline \multicolumn{8}{|c|}{ Holotrich sp., $\times 10^{5}$ cell $/ \mathrm{mL}$} \\
\hline $0 \mathrm{~h}$ & 0.63 & 0.57 & 0.40 & 0.72 & 0.27 & 0.28 & 0.74 \\
\hline $4 \mathrm{~h}$ & 0.50 & 0.75 & 0.57 & 1.07 & 1.15 & 0.45 & 0.34 \\
\hline Mean & 0.56 & 0.66 & 0.49 & 0.90 & 0.72 & 0.21 & 0.44 \\
\hline \multicolumn{8}{|c|}{ Entodiniomorphs sp., $\times 10^{6}$ cell $/ \mathrm{mL}$} \\
\hline $0 \mathrm{~h}$ & 2.82 & 2.45 & 2.43 & 2.14 & 2.26 & 1.47 & 0.11 \\
\hline $4 \mathrm{~h}$ & 3.11 & 3.40 & 3.09 & 2.52 & 2.50 & 1.44 & 0.10 \\
\hline Mean & 2.96 & 2.92 & 2.76 & 2.32 & 2.39 & 1.45 & 0.13 \\
\hline
\end{tabular}

FDP $=$ untreated discarded durian peel; FDPM = treated discarded durian peel with molasses; FDPC = treated discarded durian peel with cellulase; FDPL $=$ treated discarded durian peel with $L$. case $i$ TH14; FDPML $=$ treated discarded durian peel with molasses and L. casei TH14; SEM = standard error of the mean.

\subsection{Blood Metabolites}

Dietary treatments did not affect $(p>0.05)$ blood metabolites such as glucose, pack cell volume, BUN, total protein, albumin, globulin, the albumin-to-globulin ratio, red blood cells, hemoglobin, mean corpuscular volume, mean corpuscular hemoglobin concentration, or the RBC distribution width (Table 6). FDPML had a lower BUN concentration and higher blood glucose concentration compared with FDP, FDPM, FDPC, and FDPL.

Table 6. Effects of untreated and treated discarded durian peel in TMR on blood metabolites in growing goats.

\begin{tabular}{|c|c|c|c|c|c|c|c|}
\hline \multirow{2}{*}{ Attribute } & \multicolumn{5}{|c|}{ Dietary Treatments } & \multirow{2}{*}{ SEM } & \multirow{2}{*}{$p$-Value } \\
\hline & FDP & FDPM & FDPC & FDPL & FDPML & & \\
\hline Glu, mg/dL & 68.70 & 70.00 & 69.90 & 70.00 & 70.20 & 1.68 & 0.99 \\
\hline PCV, \% & 28.40 & 28.70 & 29.30 & 29.40 & 28.20 & 0.57 & 0.51 \\
\hline BUN, mg/dL & 20.97 & 20.09 & 20.54 & 20.45 & 17.62 & 1.24 & 0.10 \\
\hline $\mathrm{TP}, \mathrm{g} / \mathrm{L}$ & 6.21 & 6.19 & 6.33 & 6.24 & 6.20 & 0.08 & 0.82 \\
\hline $\mathrm{ALB}, \mathrm{g} / \mathrm{L}$ & 3.67 & 3.68 & 3.71 & 3.69 & 3.70 & 0.03 & 0.92 \\
\hline GLB, g/L & 2.53 & 2.51 & 2.62 & 2.54 & 2.50 & 0.06 & 0.73 \\
\hline $\mathrm{A} / \mathrm{G}$ ratio & 1.46 & 1.50 & 1.44 & 1.49 & 1.50 & 0.03 & 0.61 \\
\hline $\mathrm{RBC}, 10^{6} / \mu \mathrm{L}$ & 4.39 & 4.52 & 4.68 & 4.36 & 4.17 & 0.12 & 0.05 \\
\hline $\mathrm{Hb}, \mathrm{g} / \mathrm{dL}$ & 10.60 & 10.65 & 10.92 & 10.84 & 10.87 & 0.23 & 0.67 \\
\hline $\mathrm{MCV}, \mathrm{fL}$ & 64.92 & 63.62 & 62.97 & 67.69 & 67.93 & 1.42 & 0.05 \\
\hline $\mathrm{MCHC}, \mathrm{g} / \mathrm{dL}$ & 37.28 & 37.21 & 37.27 & 36.99 & 37.11 & 0.32 & 0.96 \\
\hline RDW, \% & 29.10 & 29.20 & 29.50 & 29.12 & 29.25 & 0.29 & 0.87 \\
\hline
\end{tabular}

FDP = untreated discarded durian peel; FDPM = treated discarded durian peel with molasses; FDPC = treated discarded durian peel with cellulase; FDPL = treated discarded durian peel with L. casei TH14; FDPML = treated discarded durian peel with molasses and L. casei TH14; SEM = standard error of the mean; Glu = glucose $\mathrm{PCV}$ = pack cell volume; $\mathrm{BUN}=$ blood urea nitrogen; $\mathrm{TP}=$ total protein; $\mathrm{ALB}=$ albumin; $\mathrm{GLB}=$ globulin; $\mathrm{A} / \mathrm{G}$ ratio = albumin-to-globulin ratio; $\mathrm{RBC}=$ red blood cell; $\mathrm{Hb}=$ hemoglobin; $\mathrm{MCV}=$ mean corpuscular volume $\mathrm{MCHC}=$ mean corpuscular hemoglobin concentration; RDW = RBC distribution width. 


\subsection{Nitrogen Utilization}

Dietary treatments did not affect $(p>0.05)$ nitrogen intake, total nitrogen loss, fecal nitrogen, nitrogen absorption, or nitrogen retention but significantly affected urinary nitrogen (Table 7). Urinary nitrogen was significantly observed between FDP and FDPML, $2.58 \mathrm{~g} / \mathrm{d}$ and $2.32 \mathrm{~g} / \mathrm{d}$, respectively. Apparent nitrogen absorption expressed as \% of nitrogen intake was significant among treatments, in which FDPML had the highest apparent nitrogen absorption, $73.63 \%$ of nitrogen intake, respectively.

Table 7. Effects of untreated and treated discarded durian peel in TMR on nitrogen utilization in growing goats.

\begin{tabular}{|c|c|c|c|c|c|c|c|}
\hline \multirow{2}{*}{ Items } & \multicolumn{5}{|c|}{ Dietary Treatments } & \multirow{2}{*}{ SEM } & \multirow{2}{*}{$p$-Value } \\
\hline & FDP & FDPM & FDPC & FDPL & FDPML & & \\
\hline \multicolumn{8}{|l|}{ Balance, $\mathrm{g} / \mathrm{d}$} \\
\hline N intake & 20.03 & 18.39 & 19.31 & 19.65 & 19.03 & 0.60 & 0.41 \\
\hline Total N loss & 8.34 & 7.55 & 7.55 & 7.77 & 7.23 & 0.37 & 0.40 \\
\hline Fecal N & 5.77 & 5.27 & 5.24 & 5.25 & 4.97 & 0.15 & 0.05 \\
\hline Urine N & $2.58^{\mathrm{a}}$ & $2.28^{b}$ & $2.32^{b}$ & $2.52^{\mathrm{a}}$ & $2.32^{b}$ & 0.30 & 0.03 \\
\hline Absorbed N & 14.27 & 13.12 & 14.07 & 14.40 & 14.06 & 0.48 & 0.93 \\
\hline Retained N & 11.69 & 10.84 & 11.75 & 11.88 & 11.74 & 0.45 & 0.39 \\
\hline \multicolumn{8}{|l|}{$\%$ of $\mathrm{N}$ intake } \\
\hline Fecal N & 29.19 & 28.90 & 27.34 & 26.94 & 26.37 & 0.41 & 0.05 \\
\hline Urine N & 13.18 & 12.32 & 11.78 & 12.96 & 11.85 & 1.44 & 0.94 \\
\hline Absorbed N & $70.81^{b}$ & $71.10^{\mathrm{b}}$ & $72.66^{\mathrm{a}}$ & $73.06^{\mathrm{a}}$ & $73.63^{a}$ & 0.40 & $<0.01$ \\
\hline Retained N & 57.63 & 58.78 & 60.88 & 60.10 & 61.79 & 1.48 & 0.35 \\
\hline
\end{tabular}

FDP $=$ untreated discarded durian peel FDPM = treated discarded durian peel with molasses; FDPC $=$ treated discarded durian peel with cellulase; FDPL $=$ treated discarded durian peel with $L$. casei $\mathrm{TH} 14 ; \mathrm{FDPML}=$ treated discarded durian peel with molasses and L. casei TH14; SEM = Standard error of the mean. ${ }^{a, b}$ Means in the same row with different letters differ $(p<0.05)$.

\section{Discussion}

Using untreated and treated discarded durian peel at the same amount in the diet mainly caused a change in fiber content such as NDF and ADF content. Using untreated discarded durian peel in the diet (FDP) had a higher NDF and ADF content compared with diets containing discarded durian peel treated with molasses (FDPM), discarded durian peel treated with cellulase (FDPC), discarded durian peel treated with L. casei TH14 (FDPL) and discarded durian peel treated with molasses and L. casei TH14 (FDPML). This was caused by the higher NDF and ADF content presented in untreated discarded durian peel than treated discarded durian peel. A lower NDF and ADF content in treated discarded durian peel is due to acid hydrolysis action during fermentation and cellulase activity. So et al. [7] used sugarcane bagasse treated with molasses in combination with cellulase or L. casei TH14 in TMR for dairy cows had a lower NDF content compared with the TMR diet containing untreated sugarcane bagasse. Oba and Allen [24] stated that fiber intake, ruminal fermentation, and production efficiency could be influenced by dietary NDF content and digestibility.

In this study, the intake of DM, expressed as either $\mathrm{kg} / \mathrm{d}$ or $\% \mathrm{BW}$ or $\mathrm{g} / \mathrm{kg} \mathrm{BW}^{0.75}$, was not significant among treatments. Thus, using untreated or treated discarded durian peel with additives at 25\% DM in the diet did not affect the daily DM intake of growing goats. Similarly, So et al. [7] found that 50\% DM of untreated and treated sugarcane bagasse combined with additives in TMR diets did not affect the daily DM intake of dairy cows. This suggests that any roughage feeds treated with or without silage additives does not affect the DM intake in ruminants. Intake of $\mathrm{ADF}(\mathrm{g} / \mathrm{d})$ was significantly different between the FDP and FDPML treatments. The reason for this effect is not clear, although DM, OM, and NDF intake were not different among these treatments. So et al. [7] found nutrient intake was unchanged in dairy cows fed TMR containing untreated and treated sugarcane bagasse with additives. Cherdthong et al. [13] fed untreated and treated rice straw with 
molasses in combination with cellulase or L. casei TH14 to Thai Native beef cattle and found no effect on nutrient intake. Dietary treatments did not affect weight gain or BW change in growing goats. The effect of additives in combination on performance is small and unclear [25]. Addah et al. [26] compared untreated and treated whole-crop barley with inoculant combination (L. buchneri, L. plantarum, and L. casei) and found no change in weight gain in growing feedlot steers.

Using pretreatment roughage feeds with additives such as LAB, molasses, and fibrolytic enzymes in the diet has been reported to improve nutrient digestibility in the rumen $[6,7,13,27]$. Additives contribute two mechanisms during fermentation: (1) acid hydrolysis reaction and (2) direct effect of fibrolytic enzymes on polysaccharide structure, which increases feed digestion efficiency of ruminal microbes $[10,28]$. This study showed that FDPM, FDPC, FDPL, and FDPML were significantly better in terms of DM, OM, CP, NDF and ADF digestibility compared with FDP. This could be due to the pretreatment effect of molasses, cellulase, and L. casei TH14 during fermentation of carbohydrate structures, subsequently resulting in nutrient digestibility improvement. So et al. [7] reported that L. casei TH14, cellulase, and molasses in combination with treated sugarcane bagasse significantly increased OM, CP, NDF, and ADF digestibility in dairy cows fed TMR diets compared with untreated sugarcane bagasse. Zhao et al. [29] evaluated the in vitro degradability of untreated rice straw and rice straw treated with L. plantarum and molasses and found a significant improvement in DM and NDF degradability by L. plantarum and molasses in combination. FDPML significantly increased estimated ME intake expressed per kg DM intake compared with FDP. This could be due to a significantly higher OM digestibility found in FDPML than FDP. So et al. [7] similarly found that a combination of sugarcane bagasse treated with molasses and L. casei TH14 in TMR-fed dairy cows increased their estimated ME intake compared untreated sugarcane bagasse.

Ruminal pH significantly determines the normal function of microbes in the rumen [30-33]. The normal ruminal $\mathrm{pH}$ ranges from 5.5 to 7.0 [34]. This study showed that FDPML significantly decreased the mean ruminal $\mathrm{pH}$ by 0.27 compared with FDP; however, the $\mathrm{pH}$ was in a normal range (6.4 to 6.7). This could be due to the higher lactic acid and LAB population found in FDPML than FDP. In addition, a significantly higher propionate concentration in FDPML compared with FDP could contribute to a lower $\mathrm{pH}$ in FDPML compared with FDP. pHs ranging from 6.4 to 6.7 showed improved fiber digestibility (Table 3) as activity of cellulolytic bacteria slows down at a $\mathrm{pH}$ less than 6 [35]. Similarly, So et al. [7] showed that a combination of sugarcane bagasse treated with molasses and L. casei TH14 in TMR fed to dairy cows significantly decreased their ruminal $\mathrm{pH}$ by 0.07 after $4 \mathrm{~h}$ of feeding. Zhang et al. [36] revealed that whole-plant corn ensiled with complex inoculants (L. plantarum L28, Enterococcus faecium EF08, and Lactobacillus buchneri LBC136) significantly decreased ruminal $\mathrm{pH}$ by 0.21 compared with ensiled whole-plant corn without inoculants in growing-finishing cattle. Lower ruminal $\mathrm{pH}$ leads to lactic-acid-dependent acid production in the rumen and is achieved approximately 2 to $6 \mathrm{~h}$ after feeding [30]. Time after offering feed and lactic acid supply rate mainly determine the change in ruminal $\mathrm{pH}[2,37] . \mathrm{NH}_{3}-\mathrm{N}$ is a main nitrogen source for microbial synthesis in the rumen $(5 \mathrm{mg} / \mathrm{dL}$ minimum and $30 \mathrm{mg} / \mathrm{dL}$ maximum requirement) [38]. Additive-treated discarded durian peel significantly decreased mean $\mathrm{NH}_{3}-\mathrm{N}$ concentration. This could be due to the activity of LAB present in fermented discarded durian peel that affected deamination, resulting in less ruminal protein degradation and enhancing nitrogen utilization in the lower digestive tract. So et al. [7] similarly revealed that additives combined with treated sugarcane bagasse significantly decreased $\mathrm{NH}_{3}-\mathrm{N}$ concentration from 22 to $20 \mathrm{mg} / \mathrm{dL}$ after $4 \mathrm{~h}$ of feeding compared with untreated sugarcane bagasse in dairy cows. FDPML significantly increased the propionate concentration, resulting in lowering the acetate-to-propionate ratio and acetate-butyrate-to-propionate ratio compared with other treatments. Increasing the propionate concentration could explain the high nutrient digestibility (Table 3) found in FDPML. In addition, a high lactic acid concentration in FDPML may contribute to an increase in the propionate concentration as lactic acid is 
biologically converted into propionate by ruminal microbes in the rumen. Similarly, So et al. [7] showed that L. casei TH14 combined with cellulase- and molasses-treated sugarcane bagasse in TMR significantly increased the propionate concentration compared with untreated sugarcane bagasse in dairy cows. Zhang et al. [36] revealed that whole-corn plant treated with complex inoculants significantly increased the propionate concentration from $6.40 \mathrm{mmol} / \mathrm{L}$ to $8.98 \mathrm{mmol} / \mathrm{L}$ in growing-finishing cattle. Cherdthong et al. [13] similarly found that rice straw treated with L. casei TH14 and molasses fed to Thai Native beef cattle significantly increased their propionate concentration from $20.3 \mathrm{~mol} / 100 \mathrm{~mol}$ to $23.2 \mathrm{~mol} / 100 \mathrm{~mol}$ compared with untreated rice straw. Estimated $\mathrm{CH}_{4}$ production was significantly lower in FDPML. The reason could be explained by the increase in propionate concentration found in FDPML as hydrogen was used for propionate synthesis, resulting in less hydrogen available in the methanogenesis pathway of methanogen bacteria to produce $\mathrm{CH}_{4}$ as the main end-product [39]. Similarly, So et al. [7] showed that sugarcane bagasse treated with $L$. casei TH14 combined with cellulase and molasses in TMR fed to dairy cows significantly decreased $\mathrm{CH}_{4}$ production by $4 \%$. Monteiro et al. [40] tested L. plantarum as direct-fed microbial in high-producing cows and similarly found decreased in $\mathrm{CH}_{4}$ production compared with no additive treatment.

Dietary treatments did not affect bacteria, fungal zoospore, total protozoa, Holotrich sp., or Entodiniomorphs sp. populations. Ruminal bacteria favor a $\mathrm{pH}$ around 7 for optimum growth [35]; this study found the $\mathrm{pH}$ ranged from 6.44 to 6.71 , which may have contributed to the unchanged bacteria population. Similarly, Bureenok et al. [41] showed that ruzi grass ensiled with molasses or fermented juice of epiphytic lactic acid bacteria fed to cows separately or as a combination did not change ruminal bacteria. However, not all previous studies found unchanged ruminal bacteria when inoculants wer eused. Cherdthong et al. [13] found that rice straw treated with molasses and L. casei TH14 combined fed to Thai Native beef cattle significantly increased ruminal bacteria population; the changed in bacteria population may have been due to the optimum ruminal $\mathrm{pH}$ ranging from 7.0 to 7.1 for bacteria growth.

Blood metabolites including glucose, pack cell volume, blood urea nitrogen, total protein, albumin, globulin, the albumin-to-globulin ratio, red blood cell, hemoglobin, mean corpuscular volume, mean corpuscular hemoglobin concentration, RBC distribution width, white blood cells, and lymphocytes were similar among dietary treatments. This suggests that the goats were in good health and had a normal metabolism status. Blood metabolites are usually used to evaluate the nutritious plane and health status in ruminants [42,43]. As well as blood metabolites, glucose, blood urea nitrogen, total protein, and albumin concentration were commonly used to evaluate protein and carbohydrate metabolism, where the higher mean value suggests a better nutrient metabolism when these metabolites changed within a normal range [44]. Liver is the main hub where glucose, albumin, and blood urea nitrogen are synthesized $[45,46]$, and glucose and albumin concentrations were greater in goats fed FDPML. Mean glucose concentration ranged from 68.70 to $70.20 \mathrm{mg} / \mathrm{dL}$, which varied in a normal range of 50 to $75 \mathrm{mg} / \mathrm{dL}$ [47]. Blood urea nitrogen is the product of $\mathrm{NH}_{3}-\mathrm{N}$ recycling and is produced from protein degradation by ruminal microbes $[48,49]$. The lower blood urea nitrogen paralleled the lower $\mathrm{NH}_{3}-\mathrm{N}$ concentration found in goats fed FDPML.

Dietary treatments did not influence nitrogen intake, fecal nitrogen, or apparent nitrogen retention, expressed as $\mathrm{g} / \mathrm{d}$ or\% of nitrogen intake in goats. Urine nitrogen was significantly lower, at $11 \%$, in FDPC and FDPML when compared with FDP. The effect of cellulase or a combination of molasses and L. casei TH14 on urinary nitrogen reduction was unknown; it may possibly be inconsistent with retained nitrogen, as it was found to be the highest in FDPC and FDPML. Cherdthong et al. [13] showed that rice straw treated with cellulase or L. casei TH14 separately or as a combination fed to Thai Native beef cattle reduced nitrogen loss both in the urine and feces but failed to reach statistical significance. 


\section{Conclusions}

This study showed that discarded durian peel fermented with a combination molasses and L. casei TH14 (FDPML) had significantly greater nutrient digestibility and propionate concentration, while estimated methane production, the acetate-to-propionate ratio and urinary nitrogen decreased when compared with untreated discarded durian peel (FDP). Therefore, a combination treated discarded durian peel with molasses and L. casei TH14 could add $25 \%$ of dry matter to the diet for growing goats without a negative impact. Further studies should evaluate the effect of fermented discarded durian peel with an additive content of higher than $25 \%$ in the diet on feed utilization, digestibility, rumen characteristics, blood metabolites, and nitrogen balance in ruminants.

Author Contributions: N.P. and P.C. conceptualized and designed the experiment. N.P. conducted laboratory and statistical analyses. N.P. and S.S. wrote the first draft of the manuscript. A.C. and P.C. reviewed and edited the manuscript. All authors have read and agreed to the published version of the manuscript.

Funding: This study was supported by the Center of Excellence in Agriculture and Natural Resources Biotechnology (CoE-ANRB: phase 3).

Institutional Review Board Statement: The study was conducted under approval procedure no. MHESI 68014/674 of the Institutional Animal Care and Use Committee, Prince of Songkla University.

Informed Consent Statement: Not applicable.

Data Availability Statement: Not applicable.

Acknowledgments: Authors are grateful for the support from Seahorse Intertrade Company Limited, Songkhla province. Additionally, authors would like to thank the Animal Production Innovation and Management Division, Faculty of Natural Resources, Prince of Songkla University, Hat Yai campus, Songkhla, Thailand, and Research Program on the Research and Development of Winged Bean Root Utilization as Ruminant Feed (RP64-6/002), Increase Production Efficiency and Meat Quality of Native Beef and Buffalo Research Group, and Research and Graduate Studies, Khon Kaen University (KKU).

Conflicts of Interest: Authors declared that research was conducted without any conflicts of interest.

\section{References}

1. Nordin, N.; Shamsudin, R.; Azlan, A.; Effendy, M. Dry matter, moisture, ash and crude fibre content in distinct segments of 'Durian Kampung' husk. Int. J. Chem. Eng. 2017, 11, 788-792.

2. Waramit, W.; Phuangborisut, S.; Wetchagool, W.; Wetchagool, N.; Phattapanit, V. Effect of dietary substitution of durian seed starch for broken rice on productive performance in broiler. Prawarun Agric. J. 2016, 13, 145-152.

3. Nuraini, A.D.; Mahata, M.E. Improving the nutrient quality of durian (Durio zibethinus) fruit waste through fermentation by using Phanerochaete chrysosporium and Neurospora crassa for poultry diet. Int. J. Poult. Sci. 2015, 14, 354-358.

4. Khota, W.; Pholsen, S.; Higgs, D.; Cai, Y. Fermentation quality and in vitro methane production of sorghum silage prepared with cellulase and lactic acid bacteria. Asian-Australas. J. Anim. Sci. 2017, 30, 1568-1574. [CrossRef] [PubMed]

5. So, S.; Cherdthong, A.; Wanapat, M. Improving sugarcane bagasse quality as ruminant feed with Lactobacillus, cellulase, and molasses. J. Anim. Sci. Technol. 2020, 62, 648-658. [CrossRef]

6. So, S.; Cherdthong, A.; Wanapat, M.; Uriyapongson, S. Fermented sugarcane bagasse with Lactobacillus combined with cellulase and molasses promotes in vitro gas kinetics, degradability, and ruminal fermentation patterns compared to rice straw. Anim. Biotechnol. 2020, 18, 1-12. [CrossRef]

7. So, S.; Wanapat, M.; Cherdthong, A. Effect of sugarcane bagasse as industrial by-products treated with Lactobacillus casei TH14, cellulase and molasses on feed utilization, ruminal ecology and milk production of mid-lactating Holstein Friesian cows. J. Sci. Food Agric. 2021, 101, 481-4489. [CrossRef]

8. So, S.; Cherdthong, A.; Wanapat, M. Growth performances, nutrient digestibility, ruminal fermentation and energy partition of Thai native steers fed exclusive rice straw and fermented sugarcane bagasse with Lactobacillus, cellulase and molasses. J. Anim. Physiol. Anim. Nutr. 2021, 106, 45-54. [CrossRef]

9. Pholsen, S.; Khota, W.; Pang, H.; Higgs, D.; Cai, Y. Characterization and application of lactic acid bacteria for tropical silage preparation. Anim. Sci. J. 2016, 87, 1202-1211. [CrossRef] 
10. Wang, S.; Guo, G.; Li, J.; Chen, L.; Dong, Z.; Shao, T. Improvement of fermentation profile and structural carbohydrate compositions in mixed silages ensiled with fibrolytic enzymes, molasses and Lactobacillus plantarum MTD-1. Ital. J. Anim. Sci. 2018, 18, 328-335. [CrossRef]

11. Wang, S.; Li, J.; Dong, Z.; Chen, L.; Shao, T. Effect of microbial inoculants on the fermentation characteristics, nutritive value, and in vitro digestibility of various forages. Anim. Sci. J. 2019, 90, 178-188. [CrossRef] [PubMed]

12. Cherdthong, A.; Suntra, C.; Khota, W. Improving nutritive value of ensiled rice straw as influenced by Lactobacillus casei. Khon Kaen Agric. J. 2019, 47, 105-110.

13. Cherdthong, A.; Suntara, C.; Khota, W.; Wanapat, M. Feed utilization and rumen fermentation characteristics of Thai-indigenous beef cattle fed ensiled rice straw with Lactobacillus casei TH14, molasses, and cellulase enzymes. Livest. Sci. 2021, 245, 104405. [CrossRef]

14. Chen, X.Z.; Li, W.Y.; Gao, C.F.; Zhang, X.P.; Weng, B.Q.; Cai, Y.M. Silage preparation and fermentation quality of kudzu, sugarcane top and their mixture treated with lactic acid bacteria, molasses and cellulase. Anim. Sci. J. 2017, 88, 1715-1721. [CrossRef] [PubMed]

15. AOAC. Official Methods of Analyses, 15th ed.; Association of Official Analytical Chemists: Arlington, VA, USA, 1990.

16. Van Soest, P.J.; Robertson, J.B.; Lewis, B.A. Methods for dietary fiber, neutral detergent fiber, and nonstarch polysaccharides in relation to animal nutrition. J. Dairy Sci. 1991, 74, 3583-3597. [CrossRef]

17. Mertens, D.R. Creating a system for meeting the fiber requirements of dairy cows. J. Dairy Sci. 1997, 80, 1463-1481. [CrossRef]

18. NRC. Nutrient Requirements of Goats: Angora, Dairy and Meat Goats in Temperate and Tropical Countries; National Academy Press: Washington, DC, USA, 1981.

19. Bremner, J.M.; Keeney, D.R. Steam distillation methods for determination of ammonium, nitrate and nitrite. Anal. Chim. Act 1965, 32, 485-495. [CrossRef]

20. Osaki, T.Y.; Kamiya, S.; Sawamura, S.; Kai, M.; Ozawa, A. Growth inhibition of Clostridium difficile by intestinal flora of infant faeces in continuous flow culture. J. Med. Microbiol. 1994, 40, 179-187. [CrossRef]

21. Moss, A.R.; Jouany, J.P.; Newbold, J. Methane production by ruminants: Its contribution to global warming. Annales Zootech. 2000, 49, 231-2353. [CrossRef]

22. Galyean, M. Laboratory Procedures in Animal Nutrition Research; New Mexico State University: Las Cruces, NM, USA, 1989.

23. Kearl, L.C. Nutrient Requirements of Ruminants in Developing Countries; International Feedstuffs Institute, Utah State University: Logan, UT, USA, 1982.

24. Oba, M.; Allen, M.S. Effects of brown midrib 3 mutation in corn silage on productivity of dairy cows fed two concentrations of dietary neutral detergent fiber: 1. Feeding behavior and nutrient utilization. J. Dairy Sci. 2000, 83, 1333-1341. [CrossRef]

25. Kung, L., Jr.; Shaver, R.D.; Grant, R.J.; Schmidt, R.J. Silage review: Interpretation of chemical, microbial, and organoleptic components of silages. J. Dairy Sci. 2018, 101, 4020-4033. [CrossRef]

26. Addah, W.; Baah, J.; Okine, E.K.; McAllister, T.A. A third-generation esterase inoculant alters fermentation pattern and improves aerobic stability of barley silage and the efficiency of body weight gain of growing feedlot cattle. J. Anim. Sci. 2012, 90, 1541-1552. [CrossRef] [PubMed]

27. Islam, M.; Enishi, O.; Purnomoadi, A.; Higuchi, K.; Takusari, N.; Terada, F. Energy and protein utilization by goats fed Italian ryegrass silage treated with molasses, urea, cellulase or cellulase + lactic acid bacteria. Small Rumin. Res. 2001, 42, 49-60. [CrossRef]

28. Yuan, X.; Guo, G.; Wen, A.; Desta, S.T.; Wang, J.; Wang, Y.; Shao, T. The effect of different additives on the fermentation quality, in vitro digestibility and aerobic stability of a total mixed ration silage. Anim. Feed Sci. Technol. 2015, 207, 41-50. [CrossRef]

29. Zhao, J.; Dong, Z.; Li, J.; Chen, L.; Bai, Y.; Jia, Y.; Shao, T. Effects of lactic acid bacteria and molasses on fermentation dynamics, structural and nonstructural carbohydrate composition and in vitro ruminal fermentation of rice straw silage. Asian-Australas. $J$. Anim. Sci. 2019, 32, 783-791. [CrossRef] [PubMed]

30. Sari, N.F.; Ridwan, R.; Fidriyanto, R.; Astuti, W.D.; Widyastuti, Y. The Effect of probiotics on high fiber diet in rumen fermentation characteristics. In IOP Conference Series: Earth and Environmental Science; IOP Publishing: Bristol, UK, 2019; Volume 251, p. 012057.

31. Dagaew, G.; Cherdthong, A.; Wanapat, M.; So, S.; Polyorach, S. Ruminal fermentation, milk production efficiency, and nutrient digestibility of lactating dairy cows receiving fresh cassava root and solid feed-block containing high sulfur. Fermentation 2021, 7, 114. [CrossRef]

32. Seankamsorn, A.; Cherdthong, A.; So, S.; Wanapat, M. Influence of chitosan sources on intake, digestibility, rumen fermentation, and milk production in tropical lactating dairy cows. Trop. Anim. Health Prod. 2021, 53, 1-9. [CrossRef]

33. Seankamsorn, A.; Cherdthong, A.; So, S.; Wanapat, M. Using glycerin with chitosan extracted from shrimp residue to enhance rumen fermentation and feed use in native Thai bulls. Vet. World 2021, 14, 1158-1164. [CrossRef]

34. Dehority, B.A.; Tirabasso, P.A. Effect of feeding frequency on bacterial and fungal concentrations, $\mathrm{pH}$, and other parameters in the rumen. J. Anim. Sci. 2001, 79, 2908-2912. [CrossRef]

35. Weimer, P.J. Why don't ruminal bacteria digest cellulose faster? J. Dairy Sci. 1996, 79, 1496-1502. [CrossRef]

36. Zhang, Y.; Zhao, X.; Chen, W.; Zhou, Z.; Meng, Q.; Wu, H. Effects of adding various silage additives to whole corn crops at ensiling on performance, rumen fermentation, and serum physiological characteristics of growing-finishing cattle. Animals 2019, 9, 695. [CrossRef] [PubMed] 
37. Seo, J.K.; Kim, S.W.; Kim, M.H.; Upadhaya, S.D.; Kam, D.K.; Ha, J.K. Direct-fed microbials for ruminant animals. Asian-Australas. J. Anim. Sci. 2010, 23, 1657-1667. [CrossRef]

38. McDonald, P.; Edwards, R.A.; Greenhalgh, J.F.D.; Morgan, C.A.; Sinclair, L.A.; Wilkinson, R.G. Animal Nutrition, 7th ed.; Prentice Hall: Harlow, UK, 2012.

39. Doyle, N.; Mbandlwa, P.; Kelly, W.J.; Attwood, G.; Li, Y.; Ross, R.P.; Stanton, C.; Leahy, S. Use of lactic acid bacteria to reduce methane production in ruminants: A critical review. Front. Microbiol. 2019, 10, 2207. [CrossRef]

40. Monteiro, H.F.; Lelis, A.L.; Brandao, V.L.; Faccenda, A.; Avila, A.S.; Arce-Cordero, J.; Silva, L.G.; Dai, X.; Restelatto, R.; Carvalho, P.; et al. In vitro evaluation of Lactobacillus plantarum as direct-fed microbials in high-producing dairy cows diets. Transl. Anim. Sci. 2020, 4, 214-228. [CrossRef]

41. Bureenok, S.; Suksombat, W.; Kawamoto, Y. Effects of the fermented juice of epiphytic lactic acid bacteria (FJLB) and molasses on digestibility and rumen fermentation characteristics of ruzigrass (Brachiaria ruziziensis) silages. Livest. Sci. 2011, 138, 266-271. [CrossRef]

42. Nozad, S.; Ramin, A.G.; Moghadam, G.; Asri-Rezaei, S.; Babapour, A.; Ramin, S. Relationship between blood urea, protein creatinine, triglycerides and macro-mineral concentrations with the quality and quantity of milk in dairy Holstein cows. In Veterinary Research Forum; Faculty of Veterinary Medicine, Urmia University: Urmia, Iran, 2012; Volume 3, p. 55.

43. Mohammadi, V.; Anassori, E.; Jafari, S. Measure of energy related biochemical metabolites changes during peri-partum period in Makouei breed sheep. In Veterinary Research Forum; Faculty of Veterinary Medicine, Urmia University: Urmia, Iran, 2016; Volume 7, p. 35

44. Russell, K.E.; Roussel, A.J. Evaluation of the ruminant serum chemistry profile. Vet. Clin. N. Am. Food Anim. Pract. 2007, 23, 403-426. [CrossRef]

45. Cozzi, G.; Ravarotto, L.; Gottardo, F.; Stefani, A.L.; Contiero, B.; Moro, L.; Brscic, M.; Dalvit, P. Reference values for blood parameters in Holstein dairy cows: Effects of parity, stage of lactation, and season of production. J. Dairy Sci. 2011, 94, 3895-3901. [CrossRef]

46. Chanjula, P.; Ngampongsai, W.; Wanapat, M. Effects of replacing ground corn with cassava chip in concentrate on feed intake, nutrient utilization, rumen fermentation characteristics and microbial populations in goats. Asian-Australas. J. Anim. Sci. 2007, 20, 1557-1566. [CrossRef]

47. Kaneko, J.J. Appendixes. In Clinical Biochemistry of Domestic Animals, 3rd ed.; Kaneko, J.J., Ed.; Academic Press: New York, NY, USA, 1980.

48. Chanjula, P.; Pakdeechanuan, P.; Wattanasit, S. Effects of dietary crude glycerin supplementation on nutrient digestibility, ruminal fermentation, blood metabolites, and nitrogen balance of goats. Asian-Australas. J. Anim. Sci. 2014, 27, 365-374. [CrossRef]

49. Chanjula, P.; Raungprim, T.; Yimmongkol, S.; Poonko, S.; Majarune, S.; Maitreejet, W. Effects of elevated crude glycerin concentrations on feedlot performance and carcass characteristics in finishing steers. Asian-Australas. J. Anim. Sci. 2016, 29, 80-88. [CrossRef] 